\title{
MIXED FORMULATION FOR ELASTIC PROBLEMS - EXISTENCE, APPROXIMATION, AND APPLICATIONS TO POISSON STRUCTURES
}

\author{
JULIAN ŁAWRYNOWICZ \\ Institute of Mathematics, Polish Academy of Sciences \\ Narutowicza 56, PL-90-136 Eodź, Poland \\ Chair of Solid State Physics, University of Eódź \\ Pomorska 149/153, PL-90-236 Eódź, Poland \\ E-mail: jlawryno@krysia.uni.lodz.pl \\ ALAIN LUCIEN MIGNOT \\ Laboratoire d'Analyse Numérique, Université de Rennes \\ 1, Avenue du Général Leclerc, F-35000 Rennes, France \\ LOUCAS C. PAPALOUCAS \\ Department of Mathematics, University of Athens \\ Panepistimiopolis, GR-15784 Athens, Greece \\ CLAUDE SURRY \\ École Nationale d'Ingénieurs, 58, Rue Jean Parrot \\ F-42023 Saint-Étienne, France
}

\begin{abstract}
A mixed formulation is given for elastic problems. Existence and uniqueness of the discretized problem are given for conformal continuous interpolations for the stress tensor components and for the components of the displacement vector. A counterpart of the problem is discussed in the case of an even-dimensional Euclidean space with an associated Hamiltonian vector field and the Poisson structure. For conformal interpolations of the same order the question remains open.
\end{abstract}

1991 Mathematics Subject Classification: Primary 73C35; Secondary 73C50, 58F05, 58A17. Research of the first author partially supported by the grant of the University of Łódź no. $505 / 454$

The paper is in final form and no version of it will be published elsewhere. 
1. Introduction. The elasticity problem can be formulated as follows: given $\mathbf{F}=\left(F_{i}\right)$ of class $C^{2}(\Omega), i \in 1, \ldots, n$, find a symmetric tensor $\sigma=\left\{\sigma_{i j}\right\},(i, j) \in\{1, \ldots, n\}^{2}$ and a displacement $\mathbf{u}=\left(u_{i}\right)$ so that

$$
\begin{gathered}
\sigma_{i j, j}+F_{i}=0, \\
\epsilon_{i j}=\frac{1}{2}\left(u_{i, j}+u_{j, i}\right), \\
\epsilon_{i j}=\mathcal{S}_{i j k l} \sigma_{k l}, \\
\mathbf{u}=0 \text { on } \Gamma_{u}, \\
\sigma_{i j} n_{j}=0 \text { on } \Gamma_{\sigma} .
\end{gathered}
$$

$\Omega$ is a bounded domain in the space $\mathbb{R}^{n}$ with boundary $\Gamma$ such that

$$
\Gamma_{u} \cup \Gamma_{\sigma}=\Gamma, \quad \Gamma_{u} \cap \Gamma_{\sigma}=\emptyset .
$$

$\mathcal{S}_{i j k l}$ denotes the inverse elasticity tensor having the following symmetry properties:

$$
\mathcal{S}_{i j k l}=\mathcal{S}_{k l i j}=\mathcal{S}_{j i k l} .
$$

The solution of equations (1)-(5) is a saddle point of the Reissner functional

$$
\mathcal{L}(\tau, \mathbf{v})=\frac{1}{2} \int_{\Omega} \mathcal{S}_{i j k l} \tau_{i j} \tau_{k l} d \Omega-\int_{\Omega} \tau_{i j} \epsilon_{i j}(\mathbf{v}) d \Omega+\int_{\Omega} F_{i} v_{i} d \Omega
$$

We have

$$
\mathcal{L}(\sigma, \mathbf{u})=\min _{\tau \in \Sigma} \max _{\mathbf{v} \in U} \mathcal{L}(\tau, \mathbf{v})
$$

with

$$
\begin{gathered}
\Sigma=\left\{\tau_{i j}: \tau_{i j}=\tau_{j i}, \tau_{i j} \in L^{2}(\Omega)\right\} \\
U=\left\{\mathbf{v}: \mathbf{v}=\left(v_{i}\right), v_{i} \in H^{1}(\Omega), v_{i}=0 \text { on } \Gamma_{u}\right\} .
\end{gathered}
$$

Define the bilinear forms and norms:

$$
\begin{gathered}
a^{*}(\sigma, \tau)=\int_{\Omega} \mathcal{S}_{i j k l} \sigma_{i j} \tau_{k l} d \Omega \\
b(\tau, \mathbf{v})=-\int_{\Omega} \tau_{i j} \epsilon_{i j}(\mathbf{v}) d \Omega \\
\|\tau\|_{\Sigma}^{2}=a^{*}(\tau, \tau), \quad\|\mathbf{v}\|^{2}=\int_{\Omega} v_{i, j} v_{i, j} d \Omega
\end{gathered}
$$

and the linear form

$$
L(\mathbf{v})=\int_{\Omega} F_{i} v_{i} d \Omega
$$

The solution of equations (1)-(5) satisfies the following weak formulation:

$$
\begin{gathered}
\exists !(\sigma, \mathbf{u}) \in \Sigma \times U, \\
a^{*}(\sigma, \tau)+b(\tau, \mathbf{u})=0, \quad \forall \tau \in \Sigma \\
b(\sigma, \mathbf{v})=L(\mathbf{v}), \quad \forall \mathbf{v} \in U .
\end{gathered}
$$

Existence and uniqueness is got by using the Brezzi-Babuška theorem [5]. The bilinear forms given by (6) and (7) are continuous on $\Sigma \times \Sigma$ and $\Sigma \times U$; here $a^{*}(\tau, \tau)$ is $\mathcal{Z}$-elliptic 
in the following sense: for any $\sigma \in \mathcal{Z}$ we have:

$$
\mathcal{Z}=\{\tau \in \Sigma: \forall \mathbf{v} \in U, \quad b(\tau, \mathbf{v})=0\}
$$

and there is a positive number $c$ with the property

$$
a^{*}(\tau, \tau)=\|\tau\|_{\Sigma}^{2} \geq c\|\tau\|_{\Sigma}^{2}
$$

so $\mathcal{Z}$ is the space of self-equilibrated stresses; $L(\mathbf{v})$ is continuous and linear on $U$. The system of equations (10) and (11) has a unique solution if there is a number $\beta$ such that

$$
\sup _{\tau \in \Sigma \backslash\{0\}}\left\{|b(\tau, \mathbf{v})| /\|\tau\|_{\Sigma}\right\}>\beta\|\mathbf{v}\|_{U}
$$

In order to verify (12) we have to take $\tau$ so that $\mathcal{S}_{i j k l} \tau_{k l}=\epsilon_{i j}(\mathbf{v})$ and use the Korn inequality [16].

2. Approximation methods. We choose finite dimension spaces $\Sigma_{h}$ and $U_{h}$ so that $\Sigma_{h} \subset \Sigma$ and $U_{h} \subset U$. In order to get the uniqueness of the discretized problem it is sufficient to verify (12). We give examples of approximation spaces where this is fulfilled. In the case of linear elasticity, following [15], we consider a family of meshes $\mathfrak{T}_{h}$ of $\Omega$ indexed by a parameter $h>0$, representing the maximum diameter $\rho(K)$ of the element $K \in \mathfrak{T}_{h}$. We consider the case where $K$ is a triangle with vertex $a_{i},\left[a_{i} a_{j}\right]$ being the edge of $K$. In the three following cases, we have (12) satisfied for $\tau_{h} \in \Sigma_{h}, \mathbf{v}_{h} \in U_{h}$.

Case 1. We have

$$
\begin{aligned}
& \Sigma_{h}^{1}=\left\{\tau_{h}: \mid \tau_{h_{i j}} \in \Sigma \cap c^{0}(\bar{\Omega}) \cap P_{2}(K), \quad \forall K \in \mathfrak{T}_{h}\right\}, \\
& U_{h}^{1}=\left\{\mathbf{v}_{h}: v_{h_{i}} \in U \cap c^{0}(\bar{\Omega}) \cap P_{1}(K), \quad \forall K \in \mathfrak{T}_{h}\right\} .
\end{aligned}
$$

Case 2. In each triangle $K$ three lines joining the middle point of each edge are drawn; $K$ is divided into four subtriangles $K_{I}, I \in(1, \ldots, 4)$ and we get $\mathfrak{T}_{h}^{\prime}$,

$$
\Sigma_{h}^{2}=\left\{\tau_{h}: \tau_{h_{i j}} \in \Sigma \cap c^{0}(\bar{\Omega}) \cap P_{1}\left(K_{I}\right), \quad \forall K_{I} \in \mathfrak{T}_{h}^{\prime}\right\}, \quad U_{h}^{2}=U_{H}^{1} .
$$

Case 3. Each triangle $K$ is divided into three subtriangles having the following vertices: the gravity center $G$ of $K$ and the three vertices of $K$, and we get

$$
\mathfrak{T}_{h}^{\prime \prime}=\cup K_{J}: \Sigma_{h}^{3}=\left\{\tau_{h}: \tau_{h_{i j}} \in \Sigma \cap c^{0}(\bar{\Omega}) \cap P_{1}\left(K_{J}\right), \quad \forall K_{J} \in \mathfrak{T}_{h}^{\prime \prime}\right\}, \quad U_{h}^{3}=U_{h}^{1} .
$$

The case of the same order of interpolation for $v_{h}$ and $\tau_{h_{i j}}$ (linear interpolation on each $K$ ) has been used in the plane elasticity. A good numerical experiment has been done by Labé [7], but we do not know whether the discretized Brezzi-Babuška condition is fulfilled in that case.

Other continuous conformal interpolations for the stresses and displacements in linear or axisymmetric elastic problems are numerically tested and give good results [1] with the same restriction about the Brezzi-Babuška condition, using quadrangle elements. In those works the meshes are quite uniform and regular. The cases can be then extended to three-dimensional elastic problems using tetrahedron or cube elements.

In order to solve the theoretical question of the uniqueness of the discretized problem (especially when we do not know whether the condition (12) is fulfilled), stabilized Galerkin finite element methods are used where continuous conformal interpolation of the same order on each element of $\mathcal{G}_{h}$ for stresses and components of the displacements 
are needed [14]. This kind of method is more close to extremal approximations of the spaces $\Sigma$ and $U$ by using bilinear forms connected to the mesh, taking in account, in the sense of least squares, some linear equations on each element (e.g., equilibrium equations connected with the behaviour law).

3. Extension to Poisson manifolds. The approximation methods described in Sect. 2, because of their reference to triangulation, can naturally be extended to the Poisson manifolds $[12,17,18]$, that is, smooth manifolds $M$ with a Poisson structure defined by a Poisson bracket given locally by the equality

$$
\{f, g\}=\sum_{\alpha=1}^{r}\left[\left(\partial / \partial q_{\alpha}\right) f\left(\partial / \partial p_{\alpha}\right) g-\left(\partial / \partial p_{\alpha}\right) f\left(\partial / \partial q_{\alpha}\right) g\right]
$$

where $f$ and $g$ are smooth real-valued functions in a co-ordinate neighbourhood of $M$, while

$$
\omega=\sum_{\alpha=1}^{r} d p_{\alpha} \wedge d q_{\alpha}, \quad\left(p_{1}, \ldots, p_{r}, q_{1}, \ldots, q_{r}, z_{1}, \ldots, z_{\ell}\right) \in \mathbb{R}^{n}, \quad n=\operatorname{dim}_{\mathbb{R}} M,
$$

is a nonsingular closed 2-form. It is clear that these notions are born out by the standard examples from classical mechanics and that $\{f, g\}$ is itself a smooth real-valued function in a co-ordinate neighbourhood in question.

Suppose now that $M=\mathbb{R}^{2 r}$ with local co-ordinates $u=\left(u_{1}, \ldots, u_{2 r}\right)$ and $g$ is a smooth real-valued function on $M$. The associated Hamiltonian vector field is of the form

$$
U_{g}=\sum_{j=1}^{2 r} a^{j}(u)\left(\partial / \partial u^{j}\right)
$$

where the coefficient functions $a^{j}(u)$ which depend on $g$ have to be determined. Let $f$ be the other smooth real-valued function on $M$ appearing in (13). Then, by (14) and (15), the Poisson bracket appears to be the function

$$
\{f, g\}=\sum_{j=1}^{2 r} \sum_{\alpha=1}^{2 r}\left\{u^{j}, u^{\alpha}\right\}\left(\partial / \partial u^{j}\right) f\left(\partial / \partial u^{\alpha}\right) g
$$

The brackets appearing in

$$
J=\left\|J_{\alpha j}(u)\right\|=\left\|\left\{u^{\alpha}, u^{j}\right\}\right\|, \quad \alpha, j=1, \ldots, 2 r,
$$

form the so-called structure matrix of $M$. Relation (16) for the Poisson bracket takes the form

$$
\{f, g\}=\nabla f \cdot J \nabla g .
$$

One of the most important examples of a Poisson structure is that associated with an $r$-dimensional Lie algebra $g$ with $C_{j \alpha}^{A}, j, \alpha, A=1, \ldots, r$, being structural constants (cf. [10], p. 251) relative to a basis $\left(x_{1}, \ldots, x_{r}\right)$. Suppose that $S$ is an $r$-dimensional vector space, with co-ordinates $\mathbf{u}=\left(u_{1}, \ldots, u_{r}\right)$ determined by a basis $\underline{\omega}=\left(\omega_{1}, \ldots, \omega_{r}\right)$. If $f$ and $h$ are smooth real-valued functions defined on $S$, the corresponding Lie-Poisson brackets 
are given by

$$
\{f, h\}=\sum_{j, \alpha, A}^{r} C_{j \alpha}^{A} x_{A}\left(\partial / \partial u_{\alpha}\right) f\left(\partial / \partial u_{j}\right) h .
$$

Formula (17) has recently been found in the works of Sophus Lie. In our time it was first set forth by F.A. Berezin. Its invariant (co-ordinate free) form is

$$
\{f, g\}(h)=\phi\left(\left[d f_{h}, d g_{h}\right]\right),
$$

where $\phi$ is a point in $g^{*}$ and $d f_{h}$ is the differential of $f$ at $\phi$ considered a vector of $g=\left(g^{*}\right)^{*}$. It is clear that (17) takes now the form (16) with the linear structure functions

$$
J_{\alpha j}(\mathbf{u})=\sum_{A=1}^{r} C_{j \alpha}^{A} u_{A}, \quad \alpha, j=1, \ldots, r .
$$

In the cases when $r=1,2$, and 4 we arrive at the complex, quaternionic, and octonionic structures, respectively, and the structural constants $C_{j \alpha}^{A}$ correspond to those described in $[11,12]$.

The Poisson bracket on $K\left(g^{*}\right)$, the algebra of polynomials on the space dual to $g$, is obtained by gluing together the Poisson brackets on the orbits [6] of the coadjoint representation. According to the Darboux theorem [18], the Poisson bracket on each orbit can locally be brought to its canonical form. In a neighbourhood of a point of general position in $g^{*}$ this can be done in a consistent fashion on different orbits, namely, it is possible to choose local co-ordinates on $g^{*}$ and the bracket (17) acquires the standard form (13). Identifying the vector space $S$ with the dual $g^{*}$ of $g$, i.e. taking $\left(\omega_{1}, \ldots, \omega_{r}\right)$ to be the dual basis of $\left(x_{1}, \ldots, x_{r}\right)$, we know that, since $g$ is finite-dimensional, the gradient $\nabla f$ of every smooth function $f: g^{*} \rightarrow \mathbb{R}$ is an element of $\left(g^{*}\right)^{*}=g$. Then the Kirillov-Poisson bracket has the invariant form

$$
\{f, g\}(\mathbf{u})=\langle\mathbf{u},[\nabla f(\mathbf{u}), \nabla h(\mathbf{u})]\rangle,
$$

where $\mathbf{u}=g^{*}$ and $[$,$] is the ordinary Lie bracket on g$. Given $h: g \rightarrow \mathbb{R}$, the corresponding Hamilton equations take the form

$$
(d / d t) u_{\alpha}=\sum_{j, A=1}^{2 r} C_{j \alpha}^{A} u_{A}\left(\partial / \partial u_{j}\right) h, \quad \alpha=1, \ldots, r .
$$

Since

$$
\left\{u_{\beta}, h\right\}=\sum_{\alpha, j, A=1}^{2 r} C_{j \alpha}^{A} u_{A}\left(\partial / \partial u_{\alpha}\right) u_{\beta}\left(\partial / \partial u_{j}\right) h, \quad \text { where }\left(\partial / \partial u_{\alpha}\right) u_{\beta}= \begin{cases}0 & \text { for } \beta \neq \alpha \\ 1 & \text { for } \beta=\alpha\end{cases}
$$

where $\beta=1, \ldots, r$, i.e.,

$$
\left\{u_{\alpha}, h\right\}=\sum_{j, A=1}^{2 r} C_{j \alpha}^{A} u_{A}\left(\partial / \partial u_{j}\right) h, \quad \alpha=1, \ldots, r,
$$

then (19) is in fact the equation of motion

$$
(d / d t) u_{\alpha}=\left\{u_{\alpha}, h\right\}, \quad \alpha=1, \ldots, r,
$$


Summing up, from the Kirillov-Poisson bracket we obtain the invariant form of the equations of motion

$$
\dot{u}_{\alpha}=\left\langle\mathbf{u},\left[\nabla u_{\alpha}, \nabla h\right]\right\rangle, \quad \alpha=1, \ldots, r .
$$

The procedure can be extended in a natural way to the associated foliations [13] and connected with the problem of stability of diffusions on their leaves $[3,4,8,9]$; diffusions being considered continuous Markov processes [2]. Finally, we can interpret $\mathbf{u}=\left(u_{1}, \ldots, u_{2 r}\right)$ as a counterpart of displacement, formulate an analogue of the elasticity problem of Sect. 1 and apply the approximation methods of Sect. 2 since it is still possible to follow the triangulation procedure. Namely, the results known for Poisson manifolds (the associate problem for elliptic equations, in particular the Laplace equation) can be extended to elastic problems. This approach cases the classical vibrations of elastic systems (membranes or elastic solids which are Hamiltonian systems).

\section{References}

[1] R. Friat, Contribution à la modélisation par éléments finis du problème de contact avec frottement et de l'indentation d'un bicouche, en comportement élasto-plastique parfait, Thèse de Mécanique, Université de Nantes, 1994.

[2] M. Fukushima, Y. Oshima and M. Takeda, Dirichlet Forms and Symmetric Markov Processes, Walter de Gruyter Studies in Mathematics 19, W. de Gruyter, Berlin-New York 1994.

[3] B. Gaveau et J. Lawrynowicz, Espaces de Dirichlet invariants biholomorphes et capacités associées, Bull. Acad. Polon. Sci. Sér. Sci. Math. 30 (1982), 63-69.

[4] - - - et L. Wojtczak, Equations de Langevin generalisées dans les milieux inhomogènes, C. R. Acad. Sci. Paris Sér. I 296 (1983), 411-413.

[5] V. Girault and P.-A. Raviart, Finite Element Methods for Navier-Stokes Equations, Theory and Algorithms, Springer Series in Computational Matematics, Springer, Berlin 1986.

[6] A. A. Kirillov, Elements of the Theory of Representations, Springer, New York 1976.

[7] P. Labé, Étude de structures stratifiées en comportement élasto-plastique par des éléments finis mixtes, Thèse de Docteur Ingénieur - Université de Rennes, 1980.

[8] J. Lawrynowicz, J. Kalina and M. Okada, Foliations by complex manifolds involving the complex hessian, (a) Ninth Conf. Analytic Functions Abstracts, Lublin 1986, pp. 29-30 (abstract), (b) Inst. of Math. Polish Acad. Sci. Preprint no. 486 (1991), ii +40 pp., (c) Dissertationes Math. 331 (1994), 45 pp.

[9] — and M. Okada, Canonical diffusion and foliation involving the complex hessian, (a) Inst. of Math. Polish Acad. Sci. Preprint no. 356 (1985), ii + 10 pp., (b) Bull. Polish Acad. Sci. Math. 34 (1986), 661-667.

[10] - J. Rembieliński and F. Succi, Generalized Hurwitz maps of the type $S \times V \rightarrow W$, anti-involutions, and quantum braided Clifford algebras, this volume, 223-240.

[11] — and L. Wojtczak in cooperation with S. Koshi and O. Suzuki, Stochastical mechanics of particle systems in Clifford-analytical formulation related to Hurwitz pairs of dimension $(8,5)$, in: Deformations of Mathematical Structures II. Hurwitz-Type Structures and Applications to Surface Physics, J. Ławrynowicz (ed.), Kluwer Academic Publishers, Dordrecht-Boston-London 1994, pp. 213-262. 
[12] A. Lichnerowicz, Les variétés de Poisson et leur algèbres de Lie associées, J. Diff. Geom. 12 (1977), 253-300.

[13] - Variétés de Poisson et feuilletages, Ann. Fac. Sci. Toulouse Math. (5) 4 (1982), 195-262.

[14] G. Lube, Stabilized Galerkin finite element methods for convection dominated and incompressible flow problems, in: Numerical Analysis and Mathematical Modelling, J.K. Kowalski and A. Wakulicz (eds.), Banach Center Publications, 29 Pol. Acad. Sci. Inst. of Math., Warszawa 1994, pp. 85-104.

[15] A.-L. Mignot and C. Surry, A mixed finite element family in plane elasticity, Appl. Math. Mod. 5 (1981), 259-262.

[16] S. Nečas and I. Hlavaček, Mathematical Theory of Elastic and Elasto-Plastic Bodies - An Introduction, Elsevier, Amsterdam 1981.

[17] P. J. Olver, Applications of Lie Groups to Differential Equations, Graduate Texts in Mathematics 107, Springer, New York-Berlin-Heidelberg-Tokyo 1986.

[18] L. C. Papaloucas, Polynomial Poisson subalgebras, Bull. Soc. Sci. Lettres Łódź 45, Sér. Rech. Déform. 19 (1995), 57-64. 